\title{
ON A GENERALIZATION OF THE NOTION OF $H^{*}$-ALGEBRA ${ }^{1}$
}

\section{PARFENY P. SAWOROTNOW}

1. Introduction. An $H^{*}$-algebra of $\mathrm{W}$. Ambrose [1] has the property that the orthogonal complement of an ideal is an ideal of the same kind. The present work is an attempt to characterize an $H^{*}$ algebra in terms of this property. For this purpose it is necessary to generalize the concept of $H^{*}$-algebra by introducing so-called twosided $H^{*}$-algebras. We assume merely that there are two involutions in the algebra: right $x \rightarrow x^{r}$ and left $x \rightarrow x^{l}$ such that $(y x, z)=\left(y, z x^{r}\right)$ and $(x y, z)=\left(y, x^{l} z\right)$. It is possible to characterize two-sided $H^{*}$ algebra in terms of the above relation imposed on ideals by making some additional assumptions on the ideal annihilators. Since every simple two-sided $H^{*}$-algebra is an $H^{*}$-algebra with the same topology we have also found a new characterization of a proper $H^{*}$-algebra.

The notation is adopted essentially from $[1 ; 6]$ and $[3]$ but unlike Ambrose we do not require that ideals be closed. We shall make a distinction between a minimal ideal and a minimal closed ideal. Also it is understood that a proper ideal is not dense in whole algebra and that an idempotent is a nonzero element.

\section{Complemented and right complemented algebra. First structure} theorem.

Definition 1. Let $A$ be a Banach algebra which is a Hilbert space. We shall call $A$ a right complemented algebra (r.c.algebra) if it has the property that the orthogonal complement of every right ideal is again a right ideal. Similarly we define a left complemented algebra (l. c. algebra). We shall call an algebra complemented if it is the same time r. c. and l. c.

As an example of a right complemented algebra one can take a right $H^{*}$-algebra introduced by M. F. Smiley [6].

EXAMPLE 1. Let $\alpha$ be a positive norm-increasing bounded operator on a Hilbert space $H$ and let $A$ be the algebra of operators of the Hilbert Schmidt type on $H$. Then $A$ is a right $H^{*}$-algebra (hence a r. c. algebra) in the scalar product $(a, b)=[\alpha a, b]$, where [ , ] denote a trace scalar product of the Hilbert Schmidt operators: $[a, b]$ $=\operatorname{tr}\left(a^{*} b\right)$.

Presented to the Society, December 28, 1954; received by the editors November 30, 1955.

1 Most of the results in this paper were taken from the author's doctoral thesis, Harvard University, 1954, written under the direction of Professor L. H. Loomis. 
Definition 2. A right complemented algebra $A$ will be called proper if $r(A)=\{x \in A \mid A x=(0)\}=(0)$.

Lemma 1. The orthogonal complement $I^{p}$ of a two-sided ideal I in a proper right complemented algebra $A$ is a two-sided ideal and is identical with both the left and the right annihilator of $I$.

Proof. We know already that $I^{p}$ is a right ideal. Since $I^{p} I \subset I^{p} \cap I$ $=(0), I^{p}$ is contained in $l(I)$ which is a two-sided ideal. Hence it is sufficient to prove that $I^{p}=l(I)$. Consider $I_{1}=I \cap l(I)$, which is also a two-sided ideal. It is readily verified that $I_{1} \subset r(A)=(0)$. Thus $l(I)=I^{p}$. It follows at once that $I^{p}=r(I)$.

It is easy to see that a semi-simple r. c. algebra is proper and that every r. c. algebra is a direct sum of its radical and a semi-simple r. c. algebra. Also it is always true for a proper r. c. algebra $A$ that $l(A)$ $=(0)$.

We shall say that an element $x \in A$ is left self-adjoint if $(x y, z)$ $=(y, x z)$ holds for every $y, z \in A$. An element $e$ will be called a left projection if it is idempotent and left self-adjoint.

LEMMA 2. Let $R$ be a proper closed regular right ideal in an r. c. algebra $A$ and let $e$ be its relative identity such that $e \in R^{p}$. Then $e$ is a left projection.

Proof. Since $e$ is a relative identity we have $e x-x \in R$ for every $x \in A$. It follows that $e R=0$ and $e R^{p}=R^{p}$; if $x \in R$, then $e x \in R$ and, since $e x \in R^{p}$ also, we have $e x=0$; if $x \in R^{p}$, then $e x-x \in R^{p} \cap R$ and hence $e x-x=0, e x=x$. In particular $e^{2}=e$. Also $e$ is left self-adjoint for if we consider $x, y \in A$ and write $x=x_{1}+x_{2}, y=y_{1}+y_{2}$ with $x_{1}$, $y_{1} \in R^{p}, x_{2}, y_{2} \in R$, we have:

$$
\begin{aligned}
& (e x, y)=\left(e x_{1}+e x_{2}, y_{1}+y_{2}\right)=\left(x_{1}, y_{1}+y_{2}\right)=\left(x_{1}, y_{1}\right) . \\
& (x, e y)=\left(x_{1}+x_{2}, e y_{1}+e y_{2}\right)=\left(x_{1}+x_{2}, y_{1}\right)=\left(x_{1}, y_{1}\right) .
\end{aligned}
$$

Lemma 3. Every semi-simple r. c. algebra A contains a left projection.

Proof. Let $x$ be an element in $A$ which does not have a right quasiinverse. Let $R$ be the closure of the regular right ideal $\{x y+y \mid y \in A\}$. Then $-x$ is a relative identity of $R$. We write $-x=e+u$ with $e \in R^{p}$, $u \in R$; then one can easily check that $e$ also is a relative identity of $R$. Hence $e$ is a left projection.

Now we define the double orthogonality and the primitivity of a projection as in [1]. Also as in [1] we show that every left projection can be expressed as a finite sum of doubly orthogonal primitive left projections, and the closed right ideal $e A$, where $e$ is a projection, is a 
minimal closed ideal if and only if $e$ is primitive. From the first part of the last statement it follows that every semi-simple r. c. algebra contains a primitive left projection.

THEOREM 1. Every semi-simple r. c. algebra $A$ is a direct sum of simple r. c. algebras each of which is a two-sided ideal in $A$.

Proof. Let $e$ be a primitive left projection in $A$ and let $I$ be the smallest closed two-sided ideal containing $e$. It is easy to see that $I$ is a minimal closed two-sided ideal. Then $I^{p}$ is also a semi-simple r. c. algebra. Using Zorn's lemma we complete the proof.

This is the first structure (Wedderburn) theorem for r. c. algebras.

\section{Two-sided $H^{*}$-algebras.}

Definition 3. A Banach algebra $A$ is called a two-sided $H^{*}$-algebra if $A$ is a Hilbert space and if for every $a \in A$ there are elements $a^{l}$ and $a^{r}$ in $A$ such that $(a b, c)=\left(b, a^{l} c\right)$ and $(b a, c)=\left(b, c a^{r}\right)$ hold for every $b, c \in A$.

Theorem 2. Every proper right $H^{*}$-algebra $A$ is a two-sided $H^{*}$ algebra.

Proof. Let $x \in A$ and let $N_{1}$ be the linear space spanned by $x$. Let $M_{1}=N_{1}^{p}, M_{2}=M_{1}^{r}=\left\{y \in A \mid y^{r} \in M_{1}\right\}$ and $N_{2}=M_{2}^{p}$. Then $N_{2}$ is onedimensional. Take $u \in N_{2}$ so that $(x, x)=\left(x^{r}, u\right)$ (note that $\left(x^{r}, u\right)=0$ for all $u \in N_{2}$ is impossible). We shall show that $u=x^{l}$. Let $y, z \in A$; then $z y^{r}=\lambda x+v$ where $\lambda$ is some complex number and $v \in M_{1}$. It follows that $y z^{r}=\bar{\lambda} x^{r}+v^{r}$ with $v^{r} \in M_{2}$. Then we have: $(x y, z)=\left(x, z y^{r}\right)$ $=(x, \lambda x+v)=(x, \lambda x)=\bar{\lambda}(x, x)=\bar{\lambda}\left(x^{r}, u\right)=\left(\bar{\lambda} x^{r}, u\right)=\left(\bar{\lambda} x^{r}+v^{r}, u\right)$ $=\left(y z^{r}, u\right)=(y, u z)$.

4. Well-complemented algebra. Second structure theorem. It turns out that in order to prove the second structure theorem for (right, left) complemented algebras it is necessary to introduce a new axiom. The algebras with the new axiom will be called well-complemented.

Definition 4. A semi-simple r. c. algebra $A$ will be called right wellcomplemented (r.w.c.) if every proper right (left) ideal in $A$ has a nonzero left (right) annihilator. A c. algebra will be called wellcomplemented (w. c.) if it is r. w. c.

A two-sided $H^{*}$-algebra furnishes an example of a w. c. algebra.

Now we prove a series of lemmas which will be used to prove the second structure theorem.

Lemma 4. Let $L$ be a left ideal in a Banach algebra $A$ such that every 
member of $L$ has a right quasi-inverse. Then $L$ is contained in the radical of $A$.

Proof. The lemma follows from the fact that if $x y$ has a right quasiinverse $u$ then $y x$ has also a right quasi-inverse $v=-(y x+y u x)$.

Lemma 5. Every closed nonzero right ideal $R$ in an r. w. c. algebra $A$ contains a left projection.

Proof. Since $A$ is semi-simple $l\left(R^{p}\right)$ contains an element $x$ which does not have a right quasi-inverse. Consider the closed regular right ideal $R_{1}=$ closure of $\{x y+y \mid y \in A\}$ of which $-x$ is a relative identity. Since $x R^{p}=(0)$, we have $R^{p} \subset R_{1}$ and hence $R_{1}^{p} \subset R$. We write $-x$ $=e+u$ with $e \in R_{1}^{p}, u \in R_{1}$; then $e$ is again a relative identity of $R_{1}$. By Lemma $2 e$ is a left projection.

Lemma 6. If $e$ is a primitive idempotent in an r.w. c. algebra $A$, then the right ideal $P=e A$ is a minimal right ideal.

Proof. We know already that $P$ is a minimal closed ideal. So it is sufficient to show that if $R$ is an ideal dense in $P$ then $e \in R$. In this case we can find $x \in R$ such that $x-e$ has a right quasi-inverse $y$. Then $x y+x-e=0$ and hence $e \in R$.

Combining Lemma 6 with the technique used in [1] we prove:

Lemma 7. Let $\left\{e_{i}\right\}$ be a family of primitive idempotents in a simple algebra $A$. Let $A_{i j}=e_{i} A e_{j}$. Then:

(i) Each $A_{i i}$ is isomorphic to the complex field;

(ii) Each $A_{i j}$ is one-dimensional;

(iii) $A_{i j} A_{j k}=A_{i k}$.

We shall say that an element $x$ in a c. algebra $A$ has a left adjoint if there exists an element $x^{l} \in A$ such that $(x y, a)=\left(y, x^{l} z\right)$ holds for all $y, z \in A$.

Lemma 8. If $e$ is a primitive left projection in an r. w. c. algebra $A$ then every element in Ae has a left adjoint.

Proof. Let $x \in A e$. Consider the right ideal $R=x A=x e A$. We may assume that $e x \neq 0$ (otherwise we replace $x$ by $y=x+e$ and prove that $y$ has a left adjoint). Then since $e x e=\lambda e$ we have $e R=e A$ from which it follows that $R$ is closed. Hence $R$ contains a left projection $f=x z=x e z$, where $z$ is some element in $A$. Then $f e \neq 0$ (otherwise $0=e f=e x e z=\lambda e z$, since $e f$ is left adjoint of $f e$ ) and hence $f e=x e z e=\mu x$ or $x=1 / \mu f e$ from which follows that $x$ has a left adjoint $x^{l}=1 / \bar{\mu} e f$.

Now we are in position to prove the second structure theorem. 
TheORem 3. Every r. w. c. algebra is a two-sided $H^{*}$-algebra. In particular a simple r. w. c. algebra $A$ is isomorphic to the algebra described in Example 1.

Proof. We follow the technique used in [1] and [6]. Let $\left\{e_{i}\right\}$ be a maximal family of doubly orthogonal primitive left projections in $A$. Consider $R=\sum_{i \in J} e_{i} A$, where $J$ is the set of indices in $\left\{e_{i}\right\}$. Then $R$ is closed. If $R \neq A$ then $R^{p}$ contains a primitive left projection $e$. Then $\left(e_{i} e, e_{i} e\right)=\left(e, e_{i} e\right)=0, e_{i} e=0$ for every $i \in J$, i.e., $e$ is doubly orthogonal to every $e_{i}$, which leads to a contradiction. Thus $R=A$. Consider $L=\sum_{i \in J} A e_{i}$ and suppose $\bar{L} \neq A$. Then the right annihilator $r(L)$ is a nonzero right ideal. This simply means that there is an element $x \in A$ such that $e_{i} x=0$ for all $e_{i}$. Then for any $y \in e_{i} A$ we have $\left(e_{i} y, x\right)=\left(y, e_{i} x\right)=0$, i.e., $x$ is orthogonal to all $e_{i} A$, hence to whole $A$, which is a contradiction. Thus $L$ is dense in $A$. Let us use the notation $A_{i j}=e_{i} A e_{j}$; then $L=\sum_{i, j} A_{i j}$. It follows from Lemma 8 that every element in $A_{i j}$ has a left adjoint (in $A_{j i}$ ). So we choose the matrix units $e_{i j} \in A_{i j}$ such that $e_{i i}=e_{i}$ and $e_{i j}^{l}=e_{j i}$. We define the matrix $\left(\alpha_{i j}\right)$ by setting $\alpha_{i j}=\left(e_{k i}, e_{k j}\right)$. It is easy to see that $\alpha_{i j}$ does not depend upon $k$ and that the matrix $\left(\alpha_{i j}\right)$ is self-adjoint. Any two elements in $L$ have the form $x=\sum_{i, j} x_{i j} e_{i j}$ and $y=\sum_{i, j} y_{i j} e_{i j}$, where $x_{i j}$ and $y_{i j}$ are suitable complex numbers, and the scalar product has the form:

$$
(x, y)=\sum x_{i k} \alpha_{k j} \bar{y}_{i j}=\operatorname{tr}\left(x \alpha y^{*}\right),
$$

where $\mathbf{x}, \mathbf{y}$ and $\boldsymbol{\alpha}$ here stand for matrices $\left(x_{i j}\right),\left(y_{i j}\right)$ and $\left(\alpha_{i j}\right)$ respectively.

Now we shall show that $\left(\alpha_{i j}\right)$ represents a bounded operator on $L^{2}(J)$. For this purpose let us consider the conjugate-linear mapping $T: x \rightarrow x^{l}$ restricted to $A e_{1}$, where 1 is some fixed index in $J$. Since every element in $A e_{1}$ has a left adjoint $T$ is defined everywhere on $A e_{1}$; the range of $T$ is a subset of $e_{1} A$ (in fact one can show that the range is entire $\left.e_{1} A\right)$. The graph of $T$ is closed: if $\left\langle x_{n}, x_{n}^{l}\right\rangle \rightarrow\langle x, u\rangle$, then $x_{n} \rightarrow x$ and $x_{n}^{l} \rightarrow u$, and for every $y, z \in A\left(x_{n} y, z\right) \rightarrow(x y, z)$ and $\left(y, x_{n}^{l} z\right)$ $\rightarrow(y, u z)$ from which it follows that $x^{l}=u$, and so $\langle x, u\rangle$ also belongs to the graph of $T$. From the closed graph theorem it follows that $T$ is continuous. Thus there exists a positive number $M$ such that

$$
\left(x^{l}, x^{l}\right) \leqq M(x, x)
$$

holds for all $x \in A e_{1}$.

Now there is a natural 1-1 correspondence between elements of $L^{2}(J)$ and $A e_{1}$, in which a member $x(i)$ of $L^{2}(J)$ corresponds to the element $x=\sum_{i \in J} x(i) e_{i 1}$ and $A e_{1}$. If $x(i)$ and $y(i)$ are finite sequences 
in $L^{2}(J)$ then $x^{l}=\sum_{i} \bar{x}(i) e_{1 i}, \quad y^{l}=\sum_{i} \bar{y}(i) e_{1 i} \quad$ and $\quad\left(x^{l}, y^{l}\right)$ $=\sum_{i, j} \bar{x}(i) \alpha_{i j} y(j)$. By the continuity of $T\left(x^{l}, y^{l}\right)=\sum_{i, j} \bar{x}(i) \alpha_{i j} y(j)$ holds for all $x(i), y(i)$ in $L^{2}(J)$. From $\left(^{*}\right)$ we have then $\sum_{i, j} x(i) \alpha_{i j} \bar{x}(j)$ $\leqq \alpha_{11} M \sum_{i}|x(i)|^{2}$. Completing the proof as in [6] we show that $L$ is an algebra of the type described in Example 1. Since $L$ is complete we have $L=A$. So $A$ is a left and hence a two-sided $H^{*}$-algebra.

To conclude this section we construct a complemented algebra which is not well-complemented.

EXAMPLE 2. Let $\tilde{\alpha}$ be some positive norm-increasing unbounded operator with domain dense in some Hilbert space $H$. Let $A$ be the set of all operators $a$ on $H$ such that $a \tilde{\alpha}$ is an operator of the Hilbert Schmidt type. Then $A$ is a complemented algebra in the scalar product $(a, b)=[a \tilde{\alpha}, b \tilde{\alpha}]=\operatorname{tr}\left(a \tilde{\alpha}(b \tilde{\alpha})^{*}\right)$. It is easy to see that there are two dense subsets of elements in $A$, every element of one having the left adjoint $a^{l}=a^{*}$ and every element of the other having the right adjoint $a^{r}=\tilde{\alpha}^{2} a^{*} \tilde{\alpha}^{-2}$ in $A$. It remains to show that $A$ is not wellcomplemented. Let us denote by $\tilde{A}$ the algebra of all operators of the Hilbert Schmidt type on $H$, and let $e$ be some left projection in $A$. It is easy to show that the Hilbert space $H$ can be realized as $\tilde{A} e$ and so that if $\tilde{x} \in H$ corresponds to $x \in \tilde{A} e$ and $\alpha$ is any operator such that $\alpha(x)$ is defined then $\alpha(\tilde{x})=\alpha x$. Since $\tilde{\alpha}$ is unbounded there exists an $a \in \tilde{A} e=A e$ such that $\tilde{\alpha} a$ is not of the Hilbert Schmidt type. This means that $a$ does not have the left adjoint. Then from Lemma 8 it follows that $A$ is not well-complemented.

\section{A special realization of a well-complemented algebra.}

EXAMPLE 3. Let $\lambda$ be a norm-decreasing linear transformation from a Hilbert space $H_{2}$ onto a Hilbert space $H_{1}$ which has a bounded inverse transformation $\mu$ from $H_{1}$ onto $H_{2}$. Let $A$ be the set of all Hilbert Schmidt operators $a$ from $H_{1}$ into $H_{2}$. Let us define the multiplication by $a \circ b=a \lambda b$. Then $A$ is a w. c. algebra in the scalar product $(a, b)$ $=\operatorname{tr}\left(a b^{*}\right)$. All the laws of an algebra are easily verified; $\|a b\| \leqq\|a\|\|b\|$ follows from the fact that $\lambda$ is norm-decreasing. The right and the left adjoint of an element $a \in A$ are defined by $a^{r}=\mu a^{*} \lambda^{*}$ and $a^{l}=\lambda^{*} a^{*} \mu$.

It turns out that every simple w. c. algebra $A$ is of the above form. This is shown in the next theorem.

TheOREM 4. Every simple w. c. algebra $A$ is of the form described in Example 3.

Proof. Let $\left\{e_{j}\right\}$ and $\left\{f_{k}\right\}$ be maximal families of doubly orthogonal primitive left and right projections in $A$ respectively. Then $A$ $=\sum_{j, k} e_{j} A f_{k}$. We choose $e_{i j}$ and $f_{k l}$ as in Theorem 3, such that 
$e_{j j}=e_{j}, e_{i j}^{l}=e_{j i}, f_{k k}=f_{k}, f_{k l}^{r}=f_{l k}$. Let $J=(j)$ and $K=(k)$ be the sets of indices in $\left\{e_{j}\right\}$ and $\left\{f_{k}\right\}$ respectively; let $1 \in J$ and $2 \in K$ be some fixed indices. Choose $w_{12} \in e_{1} A f_{2}$ such that $\left\|w_{12}\right\|=1$ and let $w_{j k}$ $=e_{j 1} w_{12} f_{2 k}$ for each $\langle j, k\rangle \in J \times K$. Then $w_{j k} \in e_{j} A f_{k}$ and $\left\|w_{j k}\right\|=1$. Since every $e_{j} A f_{k}$ is one-dimensional, $w_{j k}$ constitute an orthonormal base for $A$. Thus every $x \in A$ has the form $x=\sum_{j, k} x(j, k) w_{j k}$ and so the scalar product is of the form $(x, y)=\sum_{j, k} x(j, k) \bar{y}(j, k)$.

Now consider $w_{1 k} w_{j 2}$; since $w_{1 k} w_{j 2} \in e_{1} A f_{2}$ we have $w_{1 k} w_{j 2}=\lambda_{k j} w_{12}$ for some complex $\lambda_{k j}$. Multiplying both sides of the last equality with $e_{i 1}$ on the left and with $f_{2 l}$ on the right we get $w_{i k} w_{j l}=\lambda_{k j} w_{i l}$. Thus the multiplication has the form $x y=\sum_{i, k} x(i, k) w_{i k} \sum_{j, l} y(j, l) w_{j l}$ $=\sum_{i, l}\left(\sum_{k, j} x(i, k) \lambda_{k j} y(j, l)\right) w_{i} l$.

It remains to show that the matrix $\lambda_{k j}$ regarded as an operator from $H_{2}=L^{2}(J)$ into $H_{1}=L^{2}(K)$ is norm-decreasing and has an inverse. Since $w_{i k} \in e_{i} A$ we have $w_{i k}=\sum_{h} \tau_{k h} e_{i h}$ for some matrix $\left\{\tau_{k h}\right\}$ which can be shown to be independent of $i$; also $e_{i h}=\sum_{l} \mu_{h l} w_{i l}$ where $\left\{\mu_{h l}\right\}$ is independent of $i$. It is easy to show that $\left\{\mu_{h l}\right\}$ is the inverse of $\left\{\tau_{k h}\right\}$. From the other hand $\lambda_{k j} w_{i l}=w_{i k} w_{j l}=\sum_{h} \tau_{k h} e_{i h} w_{j l}=\tau_{k j} e_{i j} w_{j l}$ $=\tau_{k j} w_{i l}$ which simply means that $\lambda_{k j}=\tau_{k j}$ and that $\left\{\mu_{h l}\right\}$ is an inverse of $\left\{\lambda_{k j}\right\}$. In order to show that $\left\{\lambda_{k j}\right\}$ is norm-decreasing we consider $\alpha_{i j}=\left(e_{1 i}, e_{1 j}\right)=\left(\sum_{k} \mu_{i k} w_{1 k}, \sum_{l} \mu_{j l} w_{1 l}\right)=\sum_{k} \mu_{i k} \bar{\mu}_{j k}$ which simply means that $\alpha=\mu \mu^{*}$. From the fact that $\alpha$ is positive and norm-increasing it is easy to derive that $\mu$ is also norm-increasing. The rest of the proof follows immediately.

Thus the algebra of Example 3 is essentially a most general w. c. algebra. All w. c. algebras are obtained by considering all possible direct sums of the algebras of the form of that described in Example 3.

\section{BIBLIOGRAPHY}

1. W. Ambrose, Structure theorem for a special class of Banach algebras, Trans. Amer. Math. Soc. vol. 57 (1945) pp. 364-386.

2. N. Jacobson, The radical and semi-simplicity for arbitrary rings, Amer. J. Math. vol. 67 (1945) pp. 300-320.

3. I. Kaplansky, Dual rings, Ann. of Math. vol. 49 (1948) pp. 689-701.

4. L. H. Loomis, An introduction to harmonic analysis, New York, Van Nostrand, 1953, pp. 100-106.

5. G. W. Mackey, Commutative Banach algebra, mimeographed notes prepared by A. Blair, Harvard University, 1952.

6. M. F. Smiley, Right $H^{*}$-algebra, Proc. Amer. Math. Soc. vol. 4 (1953) pp. 1-4.

HARVARD UNIVERSITY AND

Catholic University of America 